\title{
Predicting impaired glucose metabolism in women with polycystic ovary syndrome by decision tree modelling
}

\author{
M. Möhlig • A. Flöter • J. Spranger • M. O. Weickert • \\ T. Schill • H. W. Schlösser • G. Brabant • \\ A. F. H. Pfeiffer • J. Selbig • C. Schöfl
}

Received: 6 January 2006 / Accepted: 6 July 2006 / Published online: 14 September 2006

(C) Springer-Verlag 2006

\begin{abstract}
Aims/hypothesis Polycystic ovary syndrome (PCOS) is a risk factor of type 2 diabetes. Screening for impaired glucose metabolism (IGM) with an OGTT has been recommended, but this is relatively time-consuming and
\end{abstract}

M. Möhlig $(\bowtie) \cdot J$. Spranger · M. O. Weickert • A. F. H. Pfeiffer Department of Clinical Nutrition,

German Institute of Human Nutrition Potsdam-Rehbruecke,

14558 Nuthetal, Germany

e-mail: mmoehlig@dife.de

M. Möhlig • J. Spranger • M. O. Weickert • A. F. H. Pfeiffer •

C. Schöfl

Department of Endocrinology, Diabetes and Nutrition,

Charité-University Medicine Berlin, Campus Benjamin Franklin,

Berlin, Germany

A. Flöter

Institute of Computer Science, University of Potsdam,

Potsdam, Germany

T. Schill $\cdot$ H. W. Schlösser

Department of Reproduction and Fertility,

Hannover Medical School,

Hannover, Germany

G. Brabant

Department of Gastroenterology, Hepatology and Endocrinology, Hannover Medical School,

Hannover, Germany

\section{J. Selbig}

Institute of Biochemistry and Biology, University of Potsdam,

Potsdam, Germany

J. Selbig

Max Planck Institute of Molecular Plant Physiology,

Potsdam, Germany inconvenient. Thus, a strategy that could minimise the need for an OGTT would be beneficial.

Materials and methods Consecutive PCOS patients $(n=118)$ with fasting glucose $<6.1 \mathrm{mmol} / 1$ were included in the study. Parameters derived from medical history, clinical examination and fasting blood samples were assessed by decision tree modelling for their ability to discriminate women with IGM (2-h OGTT value $\geq 7.8 \mathrm{mmol} / \mathrm{l}$ ) from those with NGT.

Results According to the OGTT results, 93 PCOS women had NGT and 25 had IGM. The best decision tree consisted of HOMA-IR, the proinsulin:insulin ratio, proinsulin, 17$\mathrm{OH}$ progesterone and the ratio of luteinising hormone: follicle-stimulating hormone. This tree identified 69 women with NGT. The remaining 49 women included all women with IGM (100\% sensitivity, $74 \%$ specificity to detect IGM). Pruning this tree to three levels still identified 53 women with NGT $(100 \%$ sensitivity, $57 \%$ specificity to detect IGM). Restricting the data matrix used for tree modelling to medical history and clinical parameters produced a tree using BMI, waist circumference and WHR. Pruning this tree to two levels separated 27 women with NGT (100\% sensitivity, 29\% specificity to detect IGM). The validity of both trees was tested by a leave- $10 \%$ out cross-validation.

Conclusions/interpretation Decision trees are useful tools for separating PCOS women with NGT from those with IGM. They can be used for stratifying the metabolic screening of PCOS women, whereby the number of OGTTs can be markedly reduced.

Keywords Decision tree - HOMA .

Impaired glucose tolerance $\cdot$ Insulin $\cdot$ Insulin resistance .

Polycystic ovary syndrome $\cdot$ Proinsulin .

Type 2 diabetes mellitus 


$\begin{array}{ll}\text { Abbreviations } \\ \text { ACTH } & \begin{array}{l}\text { adrenocorticotropin hormone } \\ \text { C-reactive protein } \\ \text { DRP }\end{array} \\ \text { HOMA } \% \text { B } & \begin{array}{l}\text { dehydroepiandrosterone sulphate } \\ \text { homeostasis model assessment of beta cell } \\ \text { function } \\ \text { homeostasis model assessment of insulin } \\ \text { resistance } \\ \text { HOMA-IR } \\ \text { homeostasis model assessment of insulin }\end{array} \\ \text { HOMA\%S } & \begin{array}{l}\text { impaired glucose metabolism (2-h OGTT } \\ \text { value } \geq 7.8 \text { mmol/l) }\end{array} \\ \text { IGM } & \begin{array}{l}\text { immunoradiometric assay } \\ \text { polycystic ovary syndrome } \\ \text { sexual hormone-binding globulin } \\ \text { thyroid-stimulating hormone }\end{array} \\ \text { PCOS } & \text { SHBG }\end{array}$

\section{Introduction}

Polycystic ovary syndrome (PCOS) is one of the most common endocrine diseases, affecting $5-10 \%$ of women of reproductive age [1]. PCOS is a heterogeneous disorder, characterised by hyperandrogenism, chronic anovulation and infertility [2-5].

A significant proportion of PCOS women suffer from insulin resistance, which appears to play a role in the aetiology of PCOS, since amelioration of insulin resistance by lifestyle or pharmacological intervention has been shown to improve hyperandrogenism and fertility [6-9]. Furthermore, insulin resistance is proposed to be a major risk factor for the development of type 2 diabetes mellitus and subsequent cardiovascular disease [10-13]. Estimates from several studies indicate that approximately $20-40 \%$ of adult and adolescent PCOS women, including both lean and obese patients, suffer from impaired glucose metabolism (IGM, defined as impaired glucose tolerance or type 2 diabetes) [14-17].

Since PCOS is a proposed risk factor for the development of type 2 diabetes, general screening of PCOS women for IGM has been recommended [13, 17-19]. Screening of high-risk populations appears reasonable for two reasons: (1) lifestyle intervention has been shown to prevent the development of type 2 diabetes in patients with IGT [20, 21]; and (2) early diagnosis and treatment of type 2 diabetes may reduce the burden of diabetes and its complications $[19,22,23]$.

Fasting glucose has been suggested as a screening test for IGM. However, several studies demonstrate that a substantial proportion of PCOS women with IGT or even type 2 diabetes show normal fasting glucose concentrations
$[14,16,17]$. It has therefore been suggested that all PCOS patients should undergo an OGTT for detection of IGM [13]. However, this procedure is time-consuming and inconvenient, which may limit its use as a general screening tool. It would therefore be useful to develop a screening strategy to identify those PCOS patients most likely to suffer from IGM to limit the number of OGTTs that need to be performed.

In the present study we prospectively evaluated 118 consecutive PCOS women. Parameters derived from medical history, clinical examination and fasting blood samples were assessed by decision tree modelling for their ability to discriminate women with IGM from those with NGT. Decision tree modelling is a supervised machine learning technique that reveals combinations of variables in the form of paths through decision trees. This technique is also called recursive partitioning and has recently been applied to medical data, e.g. for cancer diagnosis [24-26], HIV drug resistance [27], and the classification of insulinresistant subjects [28].

\section{Subjects and methods}

We prospectively studied 118 consecutive women with PCOS, who were referred to our clinic because of hirsutism, oligomenorrhoea/amenorrhoea or infertility. The study was carried out in accordance with the Declaration of Helsinki as revised in 2000 and the patients gave written informed consent. The cohort has previously been published in part [29-31]. The diagnosis of PCOS was based on (1) the presence of chronic ovulatory dysfunction, i.e. oligomenorrhoea (four cycles or fewer over the last 6 months) or amenorrhoea (no cycles in the last 6 months), and (2) clinical signs of hyperandrogenism, i.e. hirsutism or acne, or (3) laboratory findings, i.e. hyperandrogenaemia, defined as serum androgen levels (DHEAS, 17-OH progesterone, androstenedione or total testosterone) above the upper limit of normal for the respective assay, and (4) the exclusion of other disorders such as Cushing's syndrome, late-onset 21-hydroxylase deficiency, thyroid dysfunction, hyperprolactinaemia and androgen-secreting tumours. These diagnostic criteria for PCOS are consistent with the most commonly used diagnostic criteria for PCOS, often referred to as the National Institutes of Health consensus criteria [32]. BMI and WHR were calculated as described previously [30]. Insulin resistance was assessed both in terms of homeostasis model assessment of insulin sensitivity (HOMA\%S), using the HOMA-CIGMA, version 2, program, kindly provided by J. C. Levy [33, 34], and homeostasis model assessment of insulin resistance (HOMA-IR), calculated as fasting insulin (mU/l) $\times$ fasting 
glucose $(\mathrm{mmol} / \mathrm{l}) / 22.5$. Insulin secretion was assessed as HOMA\%B, again using the HOMA-CIGMA program. Blood pressure was measured three times in 5-min intervals in the sitting position; the mean of measurements two and three was used in further calculations. All women were asked to provide information on cycle length, smoking status, age and medication. None of the women took contraceptive drugs. All women were studied within the first 10 days following menstruation in the case of mild oligomenorrhoea, or at random if they suffered from severe oligomenorrhoea or amenorrhoea. Blood was sampled in the morning after an overnight fast and the samples were stored at $-20^{\circ} \mathrm{C}$ until analysis. Cushing's syndrome was excluded by a low-dose $(1 \mathrm{mg})$ overnight dexamethasone test and late-onset 21-hydroxylase deficiency by an ACTHstimulation test. All women had fasting glucose levels $<6.1 \mathrm{mmol} / \mathrm{l}$. During the 75 -g OGTT, a 2-h glucose value of $\geq 7.8 \mathrm{mmol} / \mathrm{l}$ was considered indicative of IGT, and a 2 -h value of $>11 \mathrm{mmol} / \mathrm{l}$ was classified as type 2 diabetes (WHO definition) [35]. IGT and type 2 diabetes were subsumed as IGM. The metabolic syndrome was defined according to criteria defined in the Third Report of the National Cholesterol Education Program Expert Panel on Detection, Evaluation, and Treatment of High Blood Cholesterol in Adults (NCEP ATP III) [36].

\section{Assays}

Glucose, insulin, C-reactive protein (CRP), 17-OH progesterone, luteinising hormone, follicle-stimulating hormone, oestradiol, progesterone, testosterone, dehydroepiandrosterone sulphate (DHEAS), sexual hormone binding globulin (SHBG), and androstenedione were measured as described previously [30]. Proinsulin was measured by a two-site immunoradiometric assay (IRMA), using a monoclonal proinsulin antibody (Clone 7F8, mouse anti-human proinsulin; Biotrend, Cologne, Germany) as the catcher antibody, and a radioiodinated monoclonal C-peptide antibody (clone PEP-001, mouse anti-human C-peptide; Novo-Nordisk, Bagsvaerd, Denmark) for detection. Proinsulin serum concentrations were calculated using recombinant human proinsulin (Sigma, Munich, Germany) as the standard. The intra-assay and interassay CVs were $<5$ and $<8 \%$, respectively, and the lower limit of sensitivity was $<1 \mathrm{pmol} / 1$. There was no cross-reactivity with insulin (up to 1,650 pmol/l) or with C-peptide (up to 9,934 pmol/1). Thyroid-stimulating hormone, cortisol and adrenocorticotropin hormone $(\mathrm{ACTH})$ were measured by chemiluminescence immunoassays (TSH and cortisol: Bayer Diagnostics, Fernwald, Germany, inter-assay CVs 3.6\%; ACTH: Nichols Institute Diagnostics, Bad Nauheim, Germany, inter-assay CV 8\%). HDL, LDL, total choles- terol and triglycerides were quantified using routine laboratory tests.

Statistical analysis

If not stated otherwise, mean values and SEM are reported (calculated using SPSS software, version 10.0, SPSS, Chicago, IL, USA). Two-tailed $\alpha<0.05$ was considered significant. The non-parametric Mann-Whitney $U$ test was used to analyse for differences in skewed continuous variables, while differences in normally distributed continuous variables were compared by the unpaired Student's $t$ test. Normal distribution was tested by the KolmogorovSmirnov test. Differences in frequencies were tested by the $\chi^{2}$ test.

A decision tree model was developed using the C4.5 algorithm [37], which consists of two steps: tree construction and tree pruning. Tree construction selects the best predictor variables that divide the cohort of a parent node into two child nodes. The split maximises the number of PCOS women with NGT in each child cohort (one node consists of only PCOS women with NGT). To achieve this, misclassification costs for women with IGM were set to 20 , while all other classification costs remained 1 by default. The child node becomes a parent node for a further split, and so on. The splitting continues until women in each node are either in one classification category or cannot be split further. The pruning step is necessary to avoid overfitting the data. The validity of the decision tree modelling was tested by a leave- $10 \%$-out cross-validation, yielding an absolute number of women with IGM misclassified in the course of the ten cross-validation runs, and a standard deviation of the classification error. This leave$10 \%$-out cross-validation was modelled ten times to yield a mean for the absolute number of women with IGM misclassified during the course of the ten leave-10\%-out cross-validation runs and a mean of the standard deviation. This procedure provides an unbiased statistic for evaluating the predictability of a fitted model [38]. Decision tree modelling was performed twice, once using all parameters depicted in Table 2 and again using the medical history and clinical parameters only.

In order to further reduce complexity, the trees were cut manually to the desired size in analogy to tree costcomplexity pruning [39]. This is indicated in the figures by the pruning line.

\section{Results}

The characteristics of the cohort are depicted in Table 1. All 118 subjects had fasting glucose concentrations below $6.1 \mathrm{mmol} / \mathrm{l}$. Twenty-five $(21.2 \%)$ subjects had an IGM 
Table 1 Clinical and endocrine features of the PCOS cohort $(n=118)$

\begin{tabular}{ll}
\hline Characteristic & Value \\
\hline Age (years) & $28.7 \pm 0.5$ \\
BMI (kg/m ${ }^{2}$ ) & $31.8 \pm 0.7$ \\
Fasting plasma glucose (mmol/l) & $4.6 \pm 0.06$ \\
Fasting insulin (pmol/l) & $100.9 \pm 6.1$ \\
HOMA\%S & $75.4 \pm 4.6$ \\
HOMA-IR & $3.09 \pm 0.22$ \\
HOMA\%B & $173.8 \pm 8.2$ \\
ACTH (pmol/l) & $3.96 \pm 0.22$ \\
Cortisol (nmol/l) & $333.2 \pm 13.8$ \\
Testosterone (nmol/l) & $3.4 \pm 0.14$ \\
Oestradiol (pmol/l) & $244.0 \pm 26.5$ \\
Progesterone (nmol/l) & $4.56 \pm 0.92$ \\
LH (U/l) & $8.5 \pm 0.5$ \\
LH:FSH & $1.81 \pm 0.17$ \\
DHEAS ( $\mu$ mol/l) & $7.56 \pm 0.27$ \\
Androstenedione (nmol/l) & $8.28 \pm 0.28$ \\
SHBG (nmol/l) & $50.8 \pm 4.1$ \\
17-OH progesterone (nmol/l) & $2.39 \pm 0.14$ \\
Overweight/obese subjects $\left(\mathrm{BMI}>25 \mathrm{~kg} / \mathrm{m}^{2}\right)$ & $90(76.3 \%)$ \\
\hline
\end{tabular}

Results are presented as means \pm SEM

FSH Follicle-stimulating hormone, $L H$ luteinising hormone

(OGTT 2-h glucose concentration $\geq 7.8 \mathrm{mmol} / \mathrm{l}$ ). Twenty women $(16.9 \%)$ had IGT and five suffered from type 2 diabetes (4.2\%). The women with IGM differed from those with NGT in that they were more obese, more insulinresistant (higher fasting insulin levels, lower HOMA\%S), had higher total testosterone, and lower SHBG levels. There were, however, no significant differences in fasting glucose, smoking status or family history of type 2 diabetes (Table 2).

Decision tree models were computed using all the parameters summarised in Table 2. Parameters of the C4.5 algorithm were set to detect IGM with a high sensitivity at the expense of specificity, since missing women with IGM is expected to have negative individual health effects. The best decision tree consecutively applied HOMA-IR, the proinsulin:insulin ratio, proinsulin, 17-OH progesterone, and the ratio of luteinising hormone:follicle-stimulating hormone (Fig. 1). This decision tree separated 69 women with NGT $(58.5 \%)$ from the remainder of the cohort (49 women), amongst them all 25 women with IGM. The sensitivity of this tree for detecting women with IGM was $100 \%$ and the specificity $74 \%$. A leave- $10 \%$-out crossvalidation was performed, which revealed a strong degree of consistency in the splitting choices. Furthermore, only 5.4 women with IGM were misclassified in the ten leave$10 \%$-out runs, yielding a mean sensitivity per leave- $10 \%$-out run of $98 \%$. The standard deviation for the misclassification was $4.6 \%$, indicating a very stable decision tree model [40]. To obtain a less complex tree, a second pruning step was performed manually. The pruning was performed after three decision levels of the tree. This pruned decision tree used HOMA-IR, the proinsulin:insulin ratio and proinsulin as predictors. This tree algorithm identified 53 women with NGT; the remaining 65 PCOS women $(55.1 \%$ of the cohort) included the 25 women with IGM. The sensitivity for the detection of women with IGM was $100 \%$ and the specificity was $57 \%$.

We further investigated the performance of decision trees that use medical history and clinical parameters only. We therefore restricted the data matrix to these parameters, which are indicated in detail in Table 2. The most suitable tree used BMI, waist circumference and WHR. This tree identified 30 PCOS women with NGT; the remainder of the cohort ( 88 women, $74.6 \%$ of the cohort) included the 25 with IGM. Again, sensitivity for detection of IGM was $100 \%$, but the specificity dropped to $32.3 \%$. Crossvalidation again yielded a strong degree of consistency and a misclassification of 3.6 women with IGM in the course of the ten leave-10\%-out runs with a standard deviation for misclassification of $5.5 \%$, which again indicates a stable tree (Fig. 2). Manual pruning of this tree to two levels separated 27 women with NGT from the remainder of the cohort (91 women, $77.2 \%$ of the cohort), which included the 25 women with IGM. Again, the sensitivity for detection of IGM was $100 \%$, and the specificity was $29 \%$.

Applying the best decision tree to a stratified screening algorithm cuts down the number of OGTTs by about $60 \%$. Pruning this tree to three levels still reduces the need for an OGTT by $45 \%$. The tree that uses clinical data alone decreases the number of OGTTs by $25 \%$. Thus, decision trees are useful tools for separating PCOS women with NGT from those with IGM.

\section{Discussion}

PCOS is one of the most common endocrine disorders in women of reproductive age, with an estimated prevalence of $5-10 \%$ among this subset of the population [2-5]. Women with PCOS often suffer from obesity, insulin resistance, IGT, or type 2 diabetes [14-17, 41]. In our cohort of 118 consecutive PCOS women with a mean age of approximately 29 years, $21 \%$ were diagnosed with either IGT or type 2 diabetes, according to the 2-h glucose concentration of an OGTT. This prevalence is similar to results reported for other PCOS cohorts of comparable age $[15,16,41]$, and is significantly higher than the IGM 
Table 2 Characteristics of the PCOS women with NGT and with IGM

\begin{tabular}{|c|c|c|c|}
\hline Characteristic & NGT $(n=93)$ & IGM $(n=25)$ & $p$ value for difference \\
\hline Age $(\text { years) })^{\mathrm{a}, \mathrm{b}}$ & $28.3 \pm 0.59$ & $30.2 \pm 0.89$ & 0.128 \\
\hline BMI $\left(\mathrm{kg} / \mathrm{m}^{2}\right)^{\mathrm{a}, \mathrm{b}}$ & $31.0 \pm 0.83$ & $34.6 \pm 1.39$ & 0.042 \\
\hline $\mathrm{WHR}^{\mathrm{ab}}$ & $0.80 \pm 0.008$ & $0.86 \pm 0.01$ & 0.001 \\
\hline Waist circumference $(\mathrm{cm})^{\mathrm{a}, \mathrm{b}}$ & $89.4 \pm 1.9$ & $101.7 \pm 3.1$ & 0.003 \\
\hline HOMА $\% \mathrm{~B}^{\mathrm{a}}$ & $168.0 \pm 9.4$ & $194.4 \pm 15.9$ & 0.182 \\
\hline Fasting plasma glucose $(\mathrm{mmol} / \mathrm{l})^{\mathrm{a}}$ & $4.57 \pm 0.07$ & $4.81 \pm 0.12$ & 0.097 \\
\hline $\mathrm{LH}(\mathrm{U} / 1)^{\mathrm{a}}$ & $8.9 \pm 0.6$ & $7.0 \pm 0.8$ & 0.109 \\
\hline DHEAS $(\mu \mathrm{mol} / 1)^{\mathrm{a}}$ & $7.29 \pm 0.27$ & $8.64 \pm 0.81$ & 0.160 \\
\hline Androstenedione $(\mathrm{nmol} / \mathrm{l})^{\mathrm{a}}$ & $8.1 \pm 0.3$ & $9.1 \pm 0.7$ & 0.152 \\
\hline Total cholesterol $(\mathrm{mmol} / \mathrm{l})^{\mathrm{a}}$ & $4.8 \pm 0.1$ & $5.0 \pm 0.3$ & 0.564 \\
\hline $\mathrm{HDL}(\mathrm{mmol} / \mathrm{l})^{\mathrm{a}}$ & $1.39 \pm 0.05$ & $1.1 \pm 0.06$ & 0.004 \\
\hline $\operatorname{LDL}(\mathrm{mmol} / \mathrm{l})^{\mathrm{a}}$ & $3.0 \pm 0.09$ & $3.3 \pm 0.22$ & 0.110 \\
\hline Triglycerides $(\mathrm{mmol} / \mathrm{l})^{\mathrm{a}}$ & $1.21 \pm 0.07$ & $2.38 \pm 0.39$ & 0.007 \\
\hline hsCRP (mg/l) & $3.56 \pm 0.56$ & $6.41 \pm 1.98$ & 0.087 \\
\hline $\mathrm{HOMA} \% \mathrm{~S}$ & $82.97 \pm 5.54$ & $48.24 \pm 3.85$ & 0.001 \\
\hline HOMA-IR & $2.79 \pm 0.23$ & $4.16 \pm 0.51$ & 0.001 \\
\hline Fasting insulin $(\mathrm{pmol} / \mathrm{l})$ & $92.77 \pm 6.78$ & $131.1 \pm 12.23$ & $<0.001$ \\
\hline Fasting proinsulin $(\mathrm{pmol} / \mathrm{l})$ & $11.44 \pm 1.72$ & $22.64 \pm 5.00$ & $<0.001$ \\
\hline Proinsulin:insulin ratio & $0.12 \pm 0.01$ & $0.16 \pm 0.02$ & 0.006 \\
\hline Testosterone (nmol/1) & $3.26 \pm 0.14$ & $3.92 \pm 0.24$ & 0.010 \\
\hline LH:FSH ratio & $1.89 \pm 0.21$ & $1.52 \pm 0.18$ & 0.444 \\
\hline Oestradiol (pmol/l) & $257.5 \pm 33.1$ & $193.1 \pm 16.7$ & 0.675 \\
\hline Progesterone (nmol/l) & $4.7 \pm 1.1$ & $3.9 \pm 1.4$ & 0.931 \\
\hline SHBG $(\mathrm{nmol} / \mathrm{l})$ & $55.93 \pm 4.94$ & $30.44 \pm 3.76$ & 0.009 \\
\hline 17-OH progesterone $(\mathrm{nmol} / \mathrm{l})$ & $2.42 \pm 0.16$ & $2.25 \pm 0.21$ & 0.693 \\
\hline Systolic BP $(\mathrm{kPa})^{\mathrm{b}}$ & $17.0 \pm 0.2$ & $17.7 \pm 0.6$ & 0.344 \\
\hline Diastolic BP $(\mathrm{kPa})^{\mathrm{b}}$ & $10.7 \pm 0.1$ & $11.6 \pm 0.4$ & 0.053 \\
\hline $\mathrm{ACTH}(\mathrm{pmol} / \mathrm{l})$ & $3.96 \pm 0.22$ & $4.62 \pm 0.44$ & 0.385 \\
\hline Cortisol (nmol/1 in response to $1 \mathrm{mg}$ dexamethasone) & $19.31 \pm 4.14$ & $26.21 \pm 8.0$ & 0.475 \\
\hline $\mathrm{TSH}(\mu \mathrm{U} / \mathrm{ml})$ & $1.73 \pm 0.14$ & $2.27 \pm 0.35$ & 0.12 \\
\hline Family history for type 2 diabetes $^{\mathrm{b}}$ & $33(35.5 \%)$ & $12(48 \%)$ & 0.30 \\
\hline Metabolic syndrome & $18(19.4 \%)$ & $12(48 \%)$ & 0.005 \\
\hline Smoker $^{\mathrm{b}}$ & $6(6.5 \%)$ & $1(4 \%)$ & 0.54 \\
\hline
\end{tabular}

Mean \pm SEM

FSH Follicle-stimulating hormone, $L H$ luteinising hormone

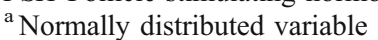

${ }^{\mathrm{b}}$ Data matrix for modelling trees using medical history and clinical examination only

prevalence in the general population [42]. Hence, screening of PCOS women for IGM has been recommended [13, 17, 19].

According to the American Diabetes Association fasting glucose is the preferred screening parameter for IGM because it is easier and faster to perform, more convenient and acceptable to patients, and less expensive than an OGTT [19]. In our group, however, there was no significant difference in fasting glucose concentrations between women with and without IGM. Thus, fasting glucose does not appear to be a suitable screening parameter in PCOS women, which is consistent with previous reports $[14,16$, 17]. Accordingly, fasting glucose was not a notch parameter in the decision trees calculated in our cohort. Therefore, an
OGTT is currently the only way to reliably detect impaired glucose metabolism in PCOS. This procedure, however, is relatively time-consuming and inconvenient for the patient, which limits its use as a general screening instrument in daily practice. Therefore, a more convenient screening strategy that minimises the need for an OGTT is desirable.

In the present study, decision tree modelling was performed to determine parameters that separate PCOS women with NGT from those with potential IGM, to identify those PCOS patients most likely to benefit from undergoing an OGTT. The best decision tree, which was based on the complete set of data, separated 69 women with NGT (58.5\% of the entire cohort); the remainder of the cohort included all women with IGM. Therefore, the 

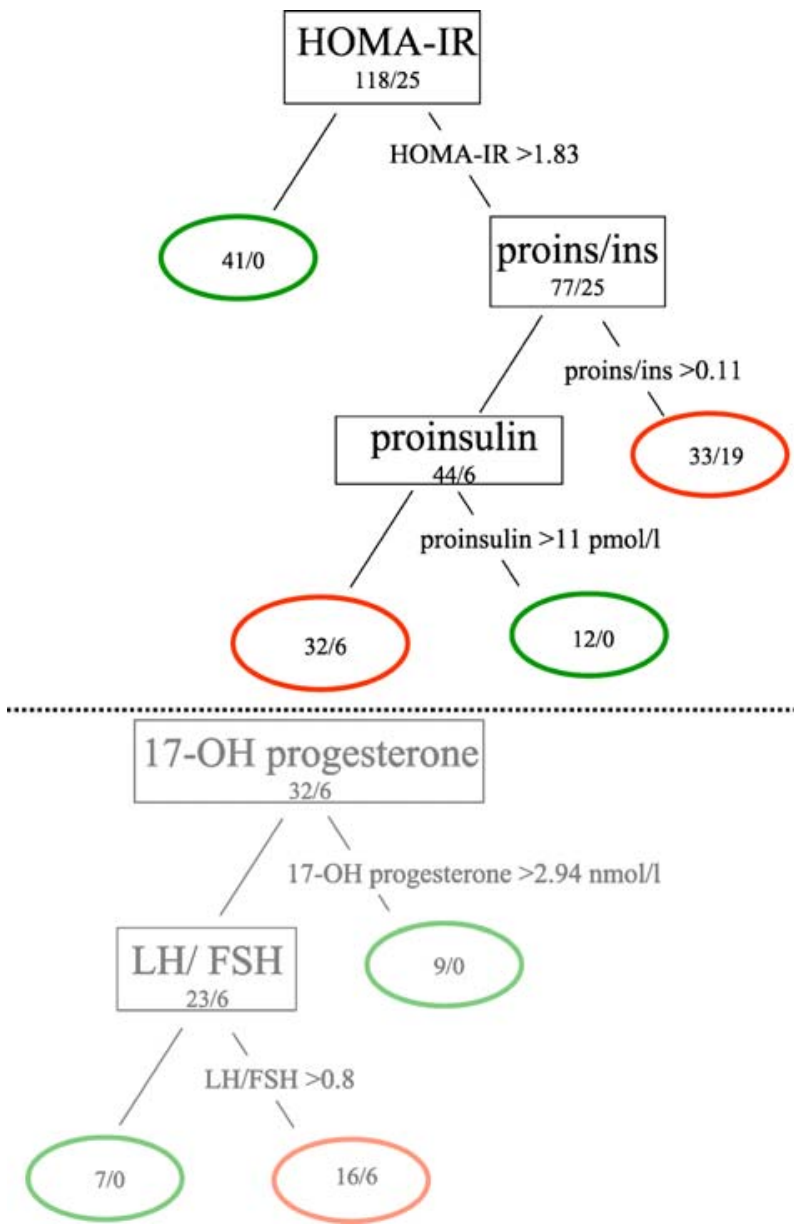

Fig. 1 The best decision tree based upon all parameters given in Table 2. The horizontal line indicates the manual pruning. $x / y$ indicates the total number of PCOS women/women with IGM. The red leaves contain women with IGM. FSH Follicle-stimulating hormone, $L H$ luteinising hormone

number of OGTTs needed to detect PCOS women with IGM could be substantially reduced from 118 to 49 . Even after pruning this tree to three levels, almost $50 \%$ of the OGTTs would be dispensable. A screening procedure using this pruned tree requires the measurement of fasting glucose, insulin and proinsulin, and the simple calculations of HOMA-IR and the proinsulin:insulin ratio. This approach appears to be feasible in daily practice. Further pruning this decision tree to the first decision node, which only requires calculation of HOMA-IR, identified 41 women with NGT. The level at which the tree is pruned may be altered according to individual needs. In general, the more decision nodes used, the better the discriminating power of the tree.

As for many biochemical and endocrine parameters, the measurement of insulin is fraught with inconsistencies [43], which also affects the calculation of HOMA-IR [44]. This problem can be overcome by extensive standardisation, but, nevertheless, it would be preferable to have a screening procedure that does not require any biochemical measure- ments. Hence, we tested decision trees based upon medical history and clinical examination only. The best of these trees pruned to two levels separated 27 women with NGT. This means that by calculating the BMI and measuring the waist circumference the number of OGTTs needed to detect all PCOS women with IGM could be reduced by about $23 \%$. This number is clearly less than the reduction that can be obtained by the aforementioned tree. Nevertheless, in clinical practice, such a low-cost and rapidly performed decision tree might be an interesting approach.

In the best decision tree, which was based on the complete, unrestricted dataset, the first decision level indicating the most important parameter for the discrimination of PCOS women with NGT from those with IGM was HOMA-IR, a parameter describing insulin resistance. The second and third decision nodes use the proinsulin: insulin ratio and proinsulin. These two parameters have been postulated to be indicators for altered beta cell function in the context of insulin resistance [45, 46]. From a pathophysiological point of view, insulin resistance and beta cell dysfunction are both key factors for the development of IGM and type 2 diabetes. Thus, it is comprehensible that the addition of parameters for altered beta cell function to a parameter of insulin resistance further enhances the discriminatory power of the decision tree.

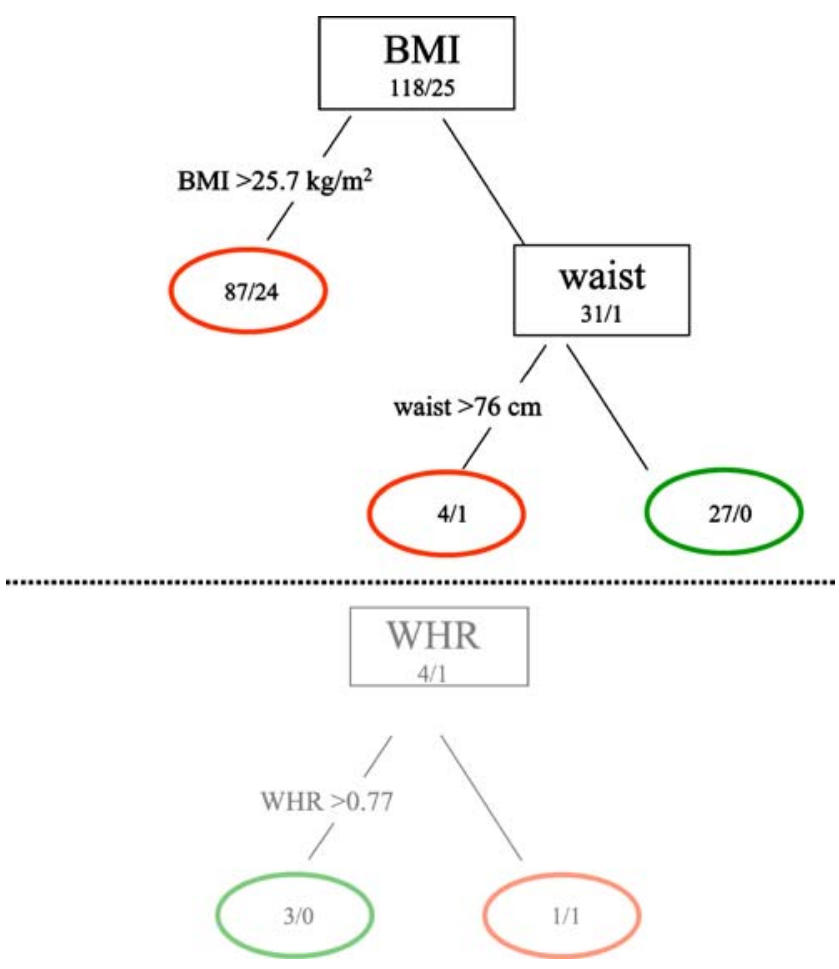

Fig. 2 The best decision tree based upon medical history and clinical examination parameters labelled with a superscript ' $b$ ' in Table 2. The horizontal line indicates the manual pruning. $x / y$ indicates the total number of PCOS women/women with IGM. The red leaves contain women with IGM 
BMI has recently been described as a predictor of insulin resistance [28]. Therefore, it is in good accordance that in the tree restricted to parameters derived from the medical history and clinical examination, BMI was the most important discriminator. Although BMI is well correlated to the degree of insulin resistance in general [47], it is, however, known that insulin resistance is not only observed in obese PCOS women but also occurs in a substantial proportion of lean PCOS patients as reported by us [29] and others [48]. It is therefore of no surprise that the decision tree which used the unrestricted dataset, including more direct parameters and indices of insulin resistance and altered beta cell function, was superior, and did not include BMI.

\section{Conclusion}

Simple decision trees could be useful tools for separating PCOS women with NGT from those with IGM. Dependent on individual needs, different decision trees can be chosen, which vary in their ability to reduce the number of OGTTs. A sophisticated strategy, which requires the measurement of fasting glucose, insulin and proinsulin, could reduce the number of necessary OGTTs by $50 \%$. Because of the wellknown variation in assays for insulin and proinsulin, the cut-off values used for the latter screening strategy are assumed to need adjustment [43]. Since this strategy is rather expensive, we searched for an alternative using only information derived from medical history and clinical examination. A screening strategy that uses BMI and waist circumference, which is a low-cost and rapidly performed approach, could save about $23 \%$ of the OGTTs needed. Therefore, applying such trees to the metabolic screening of PCOS women could minimise the number of OGTTs required to identify women with IGM.

Duality of interest All authors declare that there is no conflict of interest.

\section{References}

1. Azziz R, Woods KS, Reyna R, Key TJ, Knochenhauer ES, Yildiz BO (2004) The prevalence and features of the polycystic ovary syndrome in an unselected population. J Clin Endocrinol Metab 89:2745-2749

2. Dunaif A (1997) Insulin resistance and the polycystic ovary syndrome: mechanism and implications for pathogenesis. Endocr Rev 18:774-800

3. Franks S (1995) Polycystic ovary syndrome. N Engl J Med 333:853-861

4. Asuncion M, Calvo RM, San Millan JL, Sancho J, Avila S, Escobar-Morreale HF (2000) A prospective study of the prevalence of the polycystic ovary syndrome in unselected
Caucasian women from Spain. J Clin Endocrinol Metab 85:2434-2438

5. Knochenhauer ES, Key TJ, Kahsar-Miller M, Waggoner W, Boots LR, Azziz R (1998) Prevalence of the polycystic ovary syndrome in unselected black and white women of the southeastern United States: a prospective study. J Clin Endocrinol Metab 83:30783082

6. Pasquali R, Gambineri A, Biscotti D et al (2000) Effect of longterm treatment with metformin added to hypocaloric diet on body composition, fat distribution, and androgen and insulin levels in abdominally obese women with and without the polycystic ovary syndrome. J Clin Endocrinol Metab 85:2767-2774

7. Harborne L, Fleming R, Lyall H, Sattar N, Norman J (2003) Metformin or antiandrogen in the treatment of hirsutism in polycystic ovary syndrome. J Clin Endocrinol Metab 88: 4116-4123

8. Haas DA, Carr BR, Attia GR (2003) Effects of metformin on body mass index, menstrual cyclicity, and ovulation induction in women with polycystic ovary syndrome. Fertil Steril 79:469-481

9. Costello MF, Eden JA (2003) A systematic review of the reproductive system effects of metformin in patients with polycystic ovary syndrome. Fertil Steril 79:1-13

10. Ronnemaa T, Laakso M, Pyorala K, Kallio V, Puukka P (1991) High fasting plasma insulin is an indicator of coronary heart disease in non-insulin-dependent diabetic patients and nondiabetic subjects. Arterioscler Thromb 11:80-90

11. Skarfors ET, Selinus KI, Lithell HO (1991) Risk factors for developing non-insulin dependent diabetes: a 10 year follow up of men in Uppsala. BMJ 303:755-760

12. Zimmet P, Dowse G, Bennett P (1991) Hyperinsulinaemia is a predictor of non-insulin-dependent diabetes mellitus. Diabetes Metab 17:101-108

13. The Rotterdam Consensus Workshop (2004) Revised 2003 consensus on diagnostic criteria and long-term health risks related to polycystic ovary syndrome (PCOS). Hum Reprod 19:41-47

14. Ehrmann DA, Barnes RB, Rosenfield RL, Cavaghan MK, Imperial J (1999) Prevalence of impaired glucose tolerance and diabetes in women with polycystic ovary syndrome. Diabetes Care 22:141-146

15. Gambineri A, Pelusi C, Manicardi E et al (2004) Glucose intolerance in a large cohort of Mediterranean women with polycystic ovary syndrome: phenotype and associated factors. Diabetes 53:2353-2358

16. Legro RS, Kunselman AR, Dodson WC, Dunaif A (1999) Prevalence and predictors of risk for type 2 diabetes mellitus and impaired glucose tolerance in polycystic ovary syndrome: a prospective, controlled study in 254 affected women. J Clin Endocrinol Metab 84:165-169

17. Palmert MR, Gordon CM, Kartashov AI, Legro RS, Emans SJ, Dunaif A (2002) Screening for abnormal glucose tolerance in adolescents with polycystic ovary syndrome. J Clin Endocrinol Metab 87:1017-1023

18. Norman RJ, Masters L, Milner CR, Wang JX, Davies MJ (2001) Relative risk of conversion from normoglycaemia to impaired glucose tolerance or non-insulin dependent diabetes mellitus in polycystic ovarian syndrome. Hum Reprod 16:1995-1998

19. American Diabetes Association (2002) Clinical practice recommendations 2002. Diabetes Care 25 (Suppl 1):S21-S24

20. Tuomilehto J, Lindstrom J, Eriksson JG et al (2001) Prevention of type 2 diabetes mellitus by changes in lifestyle among subjects with impaired glucose tolerance. N Engl J Med 344:1343-1350

21. Knowler WC, Barrett-Connor E, Fowler SE et al (2002) Reduction in the incidence of type 2 diabetes with lifestyle intervention or metformin. N Engl J Med 346:393-403

22. UK Prospective Diabetes Study (UKPDS) Group (1998) Effect of intensive blood-glucose control with metformin on complications 
in overweight patients with type 2 diabetes (UKPDS 34). Lancet 352:854-865

23. UK Prospective Diabetes Study (UKPDS) Group (1998) Intensive blood-glucose control with sulphonylureas or insulin compared with conventional treatment and risk of complications in patients with type 2 diabetes (UKPDS 33). Lancet 352:837-853

24. Garzotto M, Beer TM, Hudson RG et al (2005) Improved detection of prostate cancer using classification and regression tree analysis. J Clin Oncol 23:4322-4329

25. Dennis JL, Hvidsten TR, Wit EC et al (2005) Markers of adenocarcinoma characteristic of the site of origin: development of a diagnostic algorithm. Clin Cancer Res 11:3766-3772

26. El-Serag HB, Mallat DB, Rabeneck L (2005) Management of the single liver nodule in a cirrhotic patient: a decision analysis model. J Clin Gastroenterol 39:152-159

27. Beerenwinkel N, Schmidt B, Walter H et al (2002) Diversity and complexity of HIV-1 drug resistance: a bioinformatics approach to predicting phenotype from genotype. Proc Natl Acad Sci USA 99:8271-8276

28. Stern SE, Williams K, Ferrannini E, DeFronzo RA, Bogardus C, Stern MP (2005) Identification of individuals with insulin resistance using routine clinical measurements. Diabetes $54: 333-339$

29. Spranger J, Möhlig M, Wegewitz U et al (2004) Adiponectin is independently associated with insulin sensitivity in women with polycystic ovary syndrome. Clin Endocrinol (Oxf) 61:738-746

30. Möhlig M, Spranger J, Osterhoff M et al. (2004) The polycystic ovary syndrome per se is not associated with increased chronic inflammation. Eur J Endocrinol 150:525-532

31. Schöfl C, Horn R, Schill T, Schlösser HW, Müller MJ, Brabant G (2002) Circulating ghrelin levels in patients with polycystic ovary syndrome. J Clin Endocrinol Metab 87:4607-4610

32. Zawadzki JK, Dunaif A (1992) Diagnostic criteria for polycystic ovary syndrome: towards a rational approach. In: Dunaif A, Givens J, Haseltine F, Merriam G (eds) Current issues in endocrinology and metabolism: polycystic ovary syndrome. Blackwell, New York, pp 377-384

33. Levy JC, Matthews DR, Hermans MP (1998) Correct homeostasis model assessment (HOMA) evaluation uses the computer program. Diabetes Care 21:2191-2192

34. Rudenski AS, Matthews DR, Levy JC, Turner RC (1991) Understanding "insulin resistance": both glucose resistance and insulin resistance are required to model human diabetes. Metabolism 40:908-917
35. World Health Organization (2002) Laboratory diagnosis and monitoring of diabetes mellitus. WHO, Geneva, pp 1-26

36. Ford ES, Giles WH, Dietz WH (2002) Prevalence of the metabolic syndrome among US adults: findings from the third National Health and Nutrition Examination Survey. JAMA 287:356-359

37. Quinlan JR (1993) C4.5 programs for machine learning. Morgan Kaufmann, San Mateo, USA

38. Breiman L, Freidman JH, Olshen RA, Stone CJ (1984) Classification and regression trees. Wadsworth International Group, Belmont, USA

39. Clark LA, Pregibon D (1997) Tree-based models. In: Venables WN, Ripley BD (eds) Modern applied statistics with S-PLUS. Springer-Verlag, New York, USA

40. Azuaje F (2003) Genomic data sampling and its effect on classification performance assessment. BMC Bioinformatics 4:5

41. Weerakiet S, Srisombut C, Bunnag P, Sangtong S, Chuangsoongnoen N, Rojanasakul A (2001) Prevalence of type 2 diabetes mellitus and impaired glucose tolerance in Asian women with polycystic ovary syndrome. Int J Gynaecol Obstet 75:177-184

42. Harris MI, Hadden WC, Knowler WC, Bennett PH (1987) Prevalence of diabetes and impaired glucose tolerance and plasma glucose levels in U.S. population aged 20-74 yr. Diabetes 36:523-534

43. Robbins DC, Andersen L, Bowsher R et al (1996) Report of the American Diabetes Association's Task Force on standardization of the insulin assay. Diabetes 45:242-256

44. Wallace TM, Levy JC, Matthews DR (2004) Use and abuse of HOMA modeling. Diabetes Care 27:1487-1495

45. Mykkanen L, Haffner SM, Hales CN, Ronnemaa T, Laakso M (1997) The relation of proinsulin, insulin, and proinsulin-toinsulin ratio to insulin sensitivity and acute insulin response in normoglycemic subjects. Diabetes 46:1990-1995

46. Pfützner A, Kunt T, Hohberg C et al (2004) Fasting intact proinsulin is a highly specific predictor of insulin resistance in type 2 diabetes. Diabetes Care 27:682-687

47. Ludvik B, Nolan JJ, Baloga J, Sacks D, Olefsky J (1995) Effect of obesity on insulin resistance in normal subjects and patients with NIDDM. Diabetes 44:1121-1125

48. Escobar-Morreale HF, Villuendas G, Botella-Carretero JI, Sancho J, San Millan JL (2003) Obesity, and not insulin resistance, is the major determinant of serum inflammatory cardiovascular risk markers in pre-menopausal women. Diabetologia 46:625-633 